\title{
Hemicraniectomía descompresiva en dos pacientes con infarto maligno de la arteria cerebral media
}

\author{
Patricio Mellado $\mathrm{T}^{\mathbf{1 , 5}}$, Luis Castillo $\mathrm{F}^{2,5}$, Manuel Campos $\mathrm{P}^{3}$, \\ Guillermo Bugedo $T^{2,5}$, Alberto Dougnac $L^{4,5}$, \\ Max Andresen $\mathbf{H}^{4,5}$. \\ Decompressive hemicraniectomy \\ for malignant middle cerebral artery \\ infarction. Report of two cases
}

Malignant middle cerebral territory infarction represents 5 to $10 \%$ of all brain infarctions. Its mortality is $80 \%$, due to brain herniation and it is not reduced by medical treatment. Decompressive hemicraniectomy reduces mortality to $12 \%$, and the subsequent quality of life of patients is acceptable. We report two male patients aged 61 and 54 years, with a malignant middle cerebral territory infarction who were treated with decompressive hemicraniectomy. After two years of follow up, both patients are self-sufficient and live at home with their families (Rev Méd Chile 2005; 133: 447-52).

(Key Words: Brain infarction; Intracranial pressure; Middle cerebral artery)

Recibido el 12 de enero, 2005. Aceptado el 24 de enero, 2005.

${ }^{1}$ Departamentos de Neurología, ${ }^{2}$ Anestesiología, ${ }^{3}$ Neurocirugía, ${ }^{4}$ Medicina Interna y ${ }^{5}$ Programa de Medicina Intensiva, Facultad de Medicina, Hospital Clínico de la Pontificia Universidad Católica de Chile. Santiago de Chile.

L a enfermedad cerebrovascular es la tercera causa de muerte y la etiología más frecuente de invalidez en el mundo ${ }^{1}$. El $80 \%$ corresponde a infartos encefálicos. En las últimas décadas se ha logrado un gran avance en el tratamiento del infarto encefálico, fundamentalmente con la creación de unidades especializadas en su tratamiento (unidades de stroke) y la trombólisis intravenosa ${ }^{2}$. El infarto maligno de la arteria cerebral media

Correspondencia a: Dr. Max Andresen H. Marcoleta 347 Santiago. Santiago, Chile. E mail: andresen@med.puc.cl
(IMACM), debido a su extensión y a la aparición de edema secundario, produce hernias cerebrales que pueden provocar la muerte en hasta $80 \%$ de los $\operatorname{casos}^{3,4}$. En los últimos años, esta elevada mortalidad ha sido reducida con el uso de técnicas quirúrgicas precoces y, lo que es más importante, con una morbilidad menor a la esperada.

A continuación presentamos dos pacientes con IMACM del hemisferio no dominante, que fueron sometidos a una hemicraniectomía descompresiva (HD). Ambos sobrevivieron y a los dos años realizan una vida autovalente en sus domicilios. 


\section{CASO 1}

Paciente diestro, de 61 años, con antecedentes de diabetes mellitus tipo II sin tratamiento. Ingresó 3 $h$ después de haber despertado con disartria severa, hemiplejia facio-braquio-crural, hemianestesia y hemianopsia izquierda. El resto del examen era normal. La tomografía computarizada (TC) mostró signos precoces de un IMACM derecho (Figura 1-A). Debido a la extensión del infarto se discutió la posibilidad de realizar una HD para evitar el enclavamiento y fallecimiento del paciente. Dada la naturaleza excepcional del procedimiento, se discutieron los riesgos y beneficios con familiares directos, quienes comprendieron $\mathrm{y}$ aceptaron la intervención. El estudio etiológico consistió en una ecografía carotídea, un ecocar- diograma transtorácico y un Holter de arritmias, los cuales resultaron normales.

A las $72 \mathrm{~h}$ evolucionó a sopor profundo asociado a rigidez de descerebración bilateral, sin midriasis. Se realizó una TC de control (Figura 1-B), siendo sometido a una HD de urgencia. La TC postquirúrgica mostró una hemorragia intraparenquimatosa frontal derecha, probablemente debido a un infarto venoso secundario a una craniectomía insuficiente (Figura 1-C). Evolucionó sin complicaciones, al cuarto día obedecía órdenes, fue extubado al sexto día y dado de alta a los 20 días con rehabilitación intensiva y aspirina (325 mg/día).

Reingresó al sexto mes para la instalación de la plaqueta ósea (almacenada en el banco de huesos), la cual se realizó sin complicaciones, siendo dado de

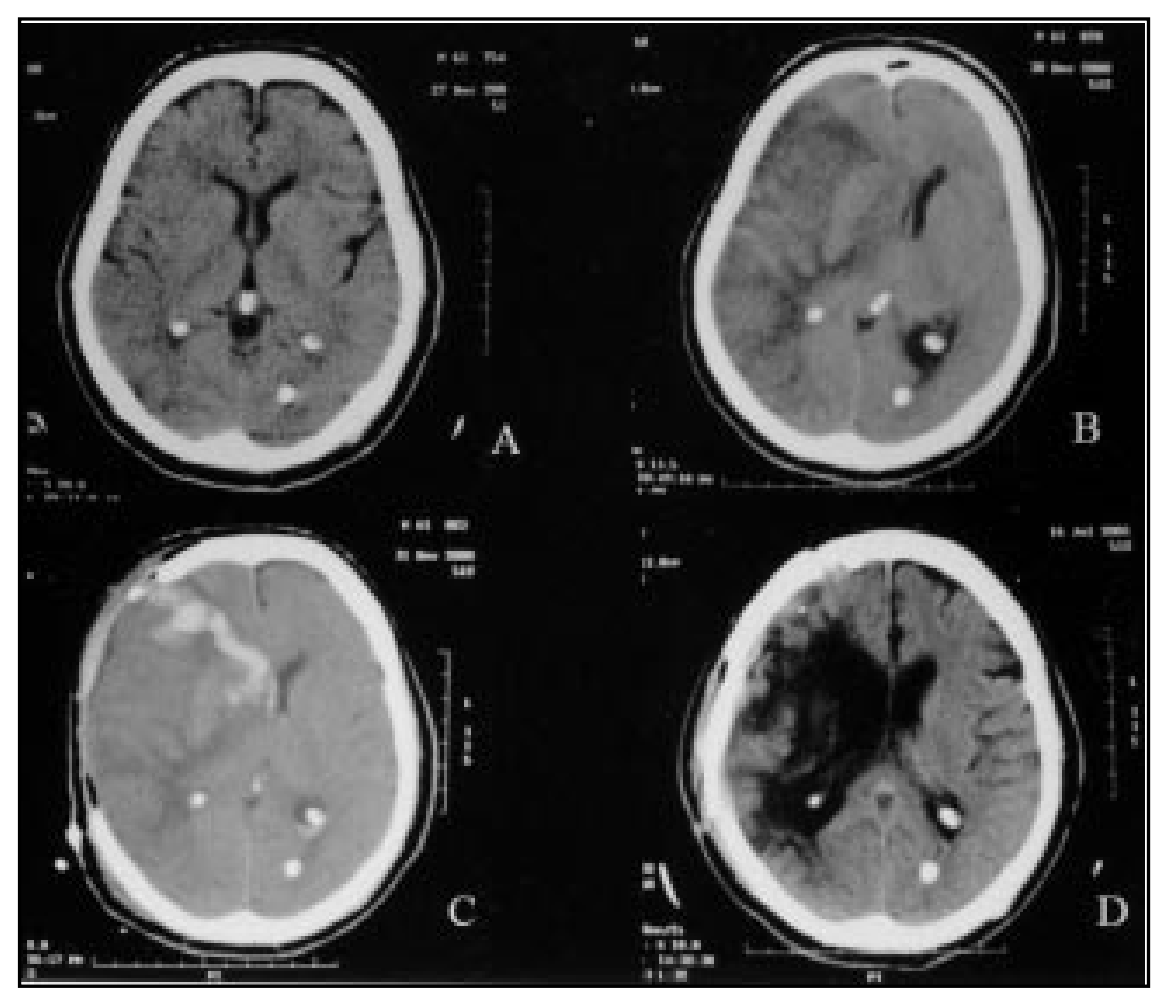

Figura 1. A) Tomografía computarizada (TC) de encéfalo al ingreso. Se observan signos precoces de un infarto de la arteria cerebral media derecha (ACMd). B) TC de control a las $72 \mathrm{~h}$. Se observa un infarto de todo el territorio de la ACMd. Además se observa una hernia subfalcina derecha y una desviación de la línea media hacia izquierda. C) TC post hemicraniectomía descompresiva (HD). Se observa una hernia encefálica a través de la hemicraniectomía y un hematoma frontal derecho, probablemente secundario a un infarto venoso debido a una compresión de venas corticales entre la hernia encefálica y el borde óseo. D) TC a los 6 meses después de la HD. Instalación de la plaqueta ósea. Se aprecia atrofia del hemisferio derecho. 
alta al octavo día (Figura 1-D). A los 6 meses presentó 2 crisis convulsivas, se indicó fenitoína (300 $\mathrm{mg} /$ día). No ha vuelto a presentar convulsiones.

A los dos años de evolución, el paciente deambula apoyado de un bastón, presenta una plejia braquial y hemianopsia izquierda. Se viste y va al baño ayudado de su familia, pero come solo (escala modificada de Rankin $=3$ ).

\section{CASO 2}

Paciente diestro, de 54 años, sin antecedentes mórbidos. Ingresó $6 \mathrm{~h}$ después de iniciar disartria severa, desviación de mirada a derecha, hemiplejia facio-braquio-crural, hemianestesia y hemianopsia izquierda. El resto de su examen no evidenciaba otras alteraciones. La TC mostró signos precoces de un IMACM derecho (Figura 2-A). Se discutió con la familia la posibilidad de una progresión a un IMACM y la eventual necesidad de una HD. La familia comprendió y aceptó la intervención.

$\mathrm{Su}$ estudio etiológico fue negativo (Tabla 1). A las $24 \mathrm{~h}$, el paciente estaba en sopor superficial sin midriasis ni aparición de Babinski a derecha. Se realizó una TC (Figura 2-B). Debido a la progresión del cuadro clínico y de la TC, se decidió realizar la $\mathrm{HD}$, la cual se efectuó sin complicaciones (Figura 2-C).

El paciente recuperó conciencia antes de $24 \mathrm{~h}$ y fue extubado a las $48 \mathrm{~h}$. No presentó complicaciones. Recuperó la movilidad de la pierna izquierda, logró deglución y fue dado de alta a los 22 días. Desde el séptimo día se inició aspirina (325 mg/día). Se mantuvo en neurorrehabilitación en su domicilio, logrando marcha en 3 meses.

Al cuarto mes se instaló la plaqueta ósea, siendo dado de alta al quinto día (Figura 2-D). A

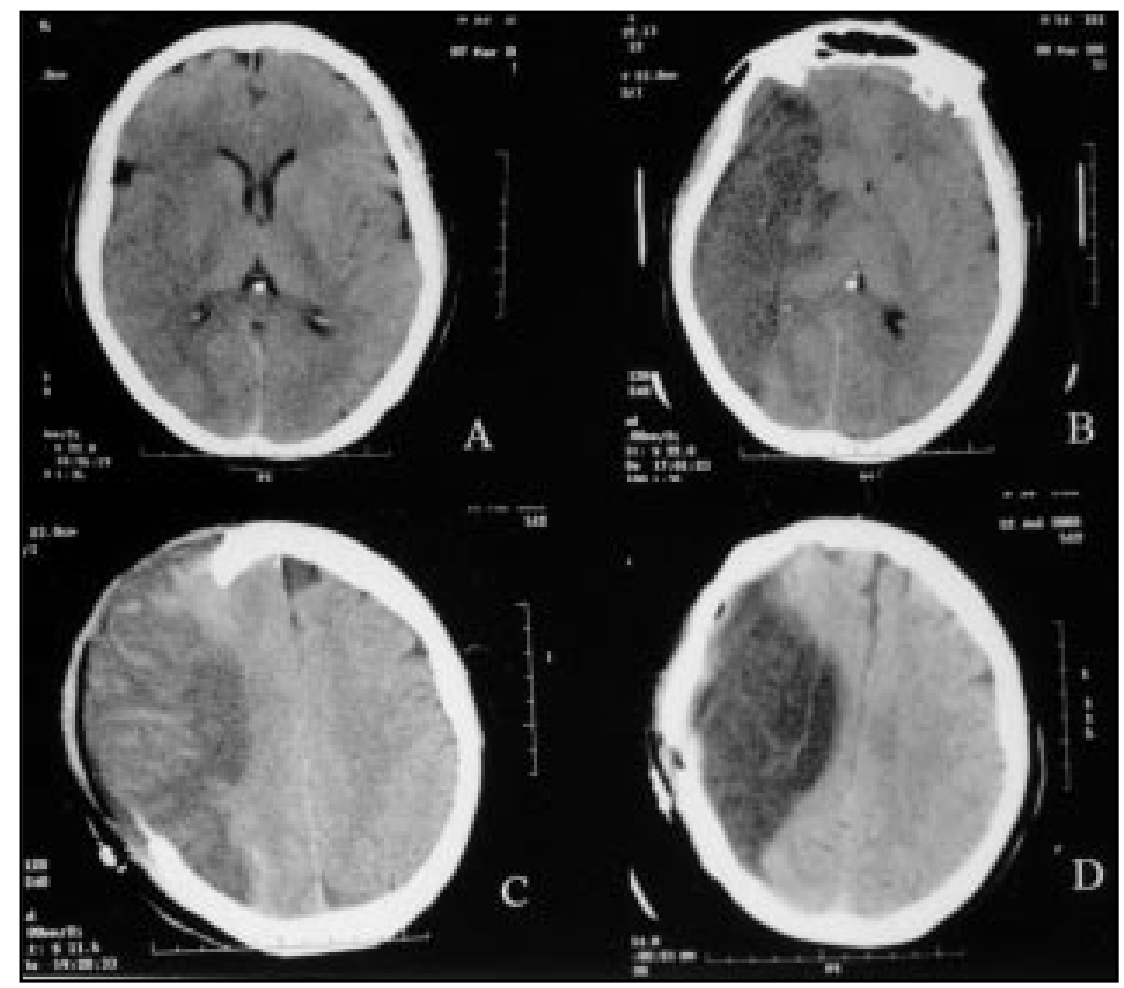

Figura 2. A) Tomografía computarizada (TC) de encéfalo al ingreso. Se observan signos precoces de un infarto de la arteria cerebral media derecha (ACMd). B) TC de control a las $24 \mathrm{~h}$. Se observa un infarto de todo el territorio de la ACMd y compresión de los ventrículos laterales. C) TC post hemicraniectomía descompresiva (HD). Se observa una hernia encefálica a través de la hemicraniectomía. D) TC a los 4 meses después de la HD. Instalación de la plaqueta ósea. 


\section{Tabla 1. Estudio etiológico del segundo paciente}

\begin{tabular}{|ll|}
\hline - & Ecografía Doppler carotídeo-vertebral \\
- & Ecocardiograma transtorácico \\
- & Holter de arritmias \\
- & Trombofilias (déficit de proteína C, S anti- \\
& trombina III, resistencia a la proteína C \\
& activada, factor V Leiden, mutación de la \\
& protrombina G20210A y homocisteína) \\
- & Síndrome antifosfolípido (anticoagulante \\
& lúpico, beta 2 glicoproteína I, anticardio- \\
& lipinas) \\
- & Angiografía por sustracción digital
\end{tabular}

los 8 meses presentó una crisis convulsiva, se inició fenitoína (300 mg/día). A los 2 años realiza una vida independiente pero limitada a su casa: camina ayudado de un bastón, come solo, se viste y va al baño ayudado por su señora (escala modificada de Rankin $=3$ ).

Al preguntarle a ambos pacientes y sus familias, individualmente, si volverían a realizar la cirugía después de haber sufrido la experiencia, respondieron afirmativamente.

\section{DisCUSIÓN}

El IMACM corresponde al infarto de al menos 2/3 del territorio de la arteria cerebral media $(\mathrm{ACM})^{3,4}$. $\mathrm{Su}$ incidencia ha sido estimada en 5 a $10 \%$ de los infartos encefálicos ${ }^{3,5,6}$. El grupo etario más afectado son los jóvenes y no existe diferencia por $\operatorname{sex}^{5}$. La causa más frecuente es la embolia de origen cardíaco o de una disección de la arteria carótida interna $(\mathrm{ACI})^{7}$. Su fisiopatología es explicada por una oclusión proximal de la arteria carótida interna o de la ACM, en ausencia de colaterales que irriguen el territorio isquémico, y además, debe existir un escaso espacio subaracnoideo, de modo tal que, cuando el edema se produzca no existirá otra opción a la de presentar hernias cerebrales ${ }^{4}$.

Las hernias cerebrales asociadas al IMACM son: subfalcina, uncal y diencefálica ${ }^{8}$. La subfalcina no tiene clínica característica, pudiendo com- primir la arteria cerebral anterior ipsilateral, lo que produce un infarto en su territorio. La hernia uncal comprime las estructuras que transcurren entre ella y la tienda del cerebelo: tercer nervio, arteria cerebral posterior (ACP) y mesencéfalo. La compresión del tercer nervio produce midriasis ipsilateral, mientras que la compresión del pedúnculo mesencefálico contralateral produce hemiparesia facio-braquio-crural y Babinski ipsilateral al hemisferio infartado (Kernohan). La compresión de la ACP produce un infarto en su territorio. Posteriormente, la compresión del mesencéfalo produce midriasis bilateral, seguida de una hernia transtentorial, la que se caracteriza por la pérdida de función neurológica en sentido rostro-caudal, finalizando con la muerte encefálica ${ }^{9}$.

Tres trabajos recientes con TC en etapas precoces de un infarto encefálico demostraron que una hipodensidad mayor a 50\% del territorio de la ACM

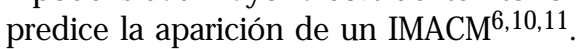

La monitorización de la presión intracraneana (PIC) en estos pacientes no ha mostrado utilidad, dado que sólo se eleva después de $24 \mathrm{~h}$ de la aparición clínica de una hernia uncal ${ }^{12,13}$. La explicación fisiopatológica de este retraso es que para que exista un desplazamiento de la línea media y por ende aparezca una hernia, se requiere de una diferencial de presiones entre dos compartimentos, pero no necesariamente que en uno de ellos la presión supere los $20 \mathrm{~mm}$ de $\mathrm{Hg}^{12}$. Así, la valoración clínica (aparición de midriasis y reflejo de Babinski ipsilateral) es trascendente.

El mejor tratamiento médico del IMACM tiene una mortalidad de hasta $80 \%$ (Tabla 2) 3 ,14-18.

\section{Tabla 2. Tratamiento médico del IM AC M}

- Hospitalización en unidades de tratamiento intensivo

Ventilación mecánica

Monitorización de presión intracraneana Monitorización metabólica (bulbo yugular, PtiO2 y midrodiálisis)

Monitorización electrofisiológica (potenciales evocados y electroencefalograma continuo)

- Uso de: manitol, glicerol, sodio hipertónico, barbitúricos e hiperventilación 
Debido a esto, se ha intentado la terapia quirúrgica, cuyo fundamento fisiopatológico es favorecer una herniación encefálica hacia fuera del cráneo y así evitar las hernias uncal y diencefálica ${ }^{19}$. La HD consiste en una craniectomía de al menos 11 a 12 $\mathrm{cm}$ de diámetro, con una extensa durotomía. No se debe realizar infartectomía, ya que no es posible determinar los límites exactos del infarto y puede extirparse parénquima encefálico viable ${ }^{20}$. La plaqueta ósea permanece en el tejido subcutáneo abdominal o en el banco de huesos. Se reinstala tres a seis meses después de la cirugía. Teóricamente, la HD puede mejorar la perfusión cerebral del área penumbra del infarto debido a que permite la irrigación de ésta por colaterales piales $^{20}$. Esto puede explicar que las secuelas observadas sean menores a las esperadas, como por ejemplo, que $2 / 3$ de los pacientes permanezcan autovalentes 21 .

El momento de realizar la HD es controversial. Si se espera la aparición de signos clínicos de una hernia cerebral, la mortalidad es de $35 \%^{22}$. Si se realiza antes de las $24 \mathrm{~h}$, basándose en la clínica y la TC, desciende a $16 \%{ }^{21}$. Por último, si se basa en la clínica y la RM, baja a $12 \%{ }^{23}$. Algunos autores sostienen que la cirugía precoz puede incluir a

\section{REFERENCIAS}

1. Wolf PA, D'Agostino RB. Epidemiology of stroke. En: Barnett HJM, Mohr JP, Stein BM, Yatsu FM. eds. Stroke: Pathophysiology, diagnosis and management. Philadelphia. Churchill Livingstone 1998; 3-28.

2. Gubitz G, SAndercock P. Extracts from "clinical evidence". Acute ischaemic stroke. BMJ 2000; 320: 692-6.

3. Hacke W, Schwab S, Horn M, Spranger M, De Georgia M, Von Kummer R. The "malignant" middle cerebral artery territory infarction: clinical course and prognostic signs. Arch Neurol 1996; 53: 309-15.

4. MeLado P, Romero C. Infarto maligno de la arteria cerebral media. En: Castillo L, Romero C, Mellado P. Cuidados Intensivos Neurológicos. Santiago. Mediterráneo 2004; 275-85. pacientes que no la requieren y que esto explica en parte el buen pronóstico ${ }^{24,25}$.

Otros trabajos han mostrado resultados menos alentadores, con una mortalidad que fluctúa entre 20 y 55\%. Además, los sobrevivientes permanecieron gravemente secuelados ${ }^{26-28}$. En la actualidad se encuentran en curso cuatro trabajos multicéntricos, prospectivos y randomizados destinados a demostrar el real beneficio de esta técnica ${ }^{4}$.

\section{CONCLUSIÓN}

Presentamos dos pacientes con un IMACM del hemisferio no dominante que fueron sometidos a una HD cuando hubo profundización del compromiso de conciencia, el cual se atribuyó a la compresión mesencefálica debido a edema hemisférico. Ambos pacientes sobrevivieron y tienen una calidad de vida aceptable.

Basados en estos dos pacientes y en la literatura disponible, pensamos que, pacientes seleccionados con un IMACM del hemisferio no dominante pueden beneficiarse de la HD. Están en curso cuatro trabajos bien diseñados destinados a demostrar la real eficacia de esta cirugía.

5. Schwarz S, Schwab S, Hacke W. Large and panhemispheric infarcts. En: Bogousslavsky J, Caplan L eds. Stroke Syndromes. Cambridge. Cambridge University Press 2001; 490-8.

6. Krieger D, Demchuk A, Kasner SE, Jauss M, Hantson L. Early clinical and radiological predictors of fatal brain swelling in ischemic stroke. Stroke 1999; 30: 287-92.

7. Heinsius T, Bogoussiavsky J, Van Meue G. Large infarcts in the middle cerebral artery territory: etiology and outcome patterns. Neurology 1998; 50: 341-50.

8. Plum F, Posner JB. The Diagnosis of Stupor and Coma. Oxford. Oxford University Press. 2000.

9. WIJDICKS EFM. Clinical diagnosis and confirmatory testing of brain death in adults. En: Wijdicks EFM ed. Brain Death. Philadelphia. Lippincott Williams Wilkins 2001; 61-90. 
10. Von Kummer R, Meyding-Lamandé U, Forsting M, Rosin L, Rieke K et al. Sensitivity and prognostic value of early CT in occlusion of the middle artery trunk. Am J Neuroradiol 1994; 15: 9-15.

11. Kasner SE, Demchuk AM, Berrouschot J, Schmutzhard E, Harms L, VerRo P et al. Predictors of fatal brain edema in massive hemispheric ischemic stroke. Stroke 2001; 32: 2117-23.

12. FRANK JL Large hemispheric infarction, deterioration, and intracranial pressure. Neurology 1995; 45: 1286-90.

13. Schwab S, Aschoff A, Spranger M, Albert F, Hacke W. The value of intracranial pressure monitoring in acute hemispheric stroke. Neurology 1996; 47: 393-8.

14. Aiyagari V, DiRInger MN. Management of lange hemispheric strokes in the neurological intensive care. The Neurologist 2002; 8: 152-62.

15. Deibert E, Diringer MN. The intensive care management of acute ischemic. Stroke 1999; 5: 313-25.

16. MAYER SA, DenNIS LJ. Management of increased intracranial pressure. The Neurologist 1998; 4: 212.

17. Sмттн WS, Aminoff MJ. Introduction to monitoring in the neurological intensive care unit and its future impact. The Neurologist 2000; 6: 83-97.

18. Schwab S, Bertram M, Hacke W. Critical care of cerebrovascular disease. En: Fisher M ed. Stroke Therapy. Boston. Butterworth Heinemann 2001; 225-59.

19. Davis SM, Donnan GA, Grotta JC, Hacke W. Surgical interventions in the treatment of acute ischemic infarction. En: Davis SM, Donnan GA, Grotta JC, Hacke W eds. Interventional therapy in acute stroke. Malden. Blackwell Science 1998; 117-29.
20. Wagner S, Schnippering H, Aschoff A, Koziol Ja, Schwab S, Steiner T. Suboptimum hemicraniectomy as a cause of additional cerebral lesions in patients with malignant infarction of the middle cerebral artery. J Neurosurg 2001; 94: 693-6.

21. Schwab S, Steiner T, Aschoff A, Schwarz S, Steiner HH ET AL. Early hemicraniectomy in patients with complete middle cerebral artery infarction. Stroke 1998; 29: 1888-93.

22. Rueke K, Schwab S, Krieger D, Von Kummer R, Aschoff A ET AL. Decompressive surgery in spaceoccupying hemispheric infarction: results of an open, prospective trial. Crit Care Med 1995; 23: 1576-87.

23. Georgiadis D, Schwarz S, Aschoff A, Schwab S. Hemicraniectomy and moderate hypothermia in patients with severe ischemic stroke. Stroke 2002; 33: 1584-8.

24. WIJDICKs EFM. Management of Massive Hemispheric Cerebral Infarct: Is there a Ray of Hope? Mayo Clin Proc 2000; 75: 945-52.

25. WiJDICKS EFM. Hemicraniectomy in massive hemispheric stroke: a stark perspective on a radical procedure. Can J Neurol Sci 2000; 27: 271-3.

26. WiJdicks EFM, DiRIngER MN. Middle Cerebral Artery Territory Infarction and Early Brain Swelling: Progression and Effect of Age on Outcome. Mayo Clin Proc 1998; 73: 829-36.

27. Кон MS, Goн KYC, Tung My, Chan C. Is decompressive craniectomy for acute cerebral infarction of any benefit. Surg Neurol 2000; 53: 225-30.

28. HoltKamp M, Buchieim $K$, Unterberg A, Hoffmann O, SchIELKE E ET AL. Hemicraniectomy in the elderly patients with space occupying media infarction: improved survival but poor functional outcome. J Neurol Neurosurg Psychiatry 2001; 70: 226-8. 